\title{
THE BOUNDEDNESS OF DEGREE OF FANO VARIETIES WITH PICARD NUMBER ONE
}

\author{
ALAN MICHAEL NADEL
}

\section{CONTENTS}

0 . Introduction

1. Rational curves

1.1. Deformation theory of morphisms

1.2. Free rational curves

1.3. The invariant $d(M)$

2. Special subvarieties in Fano varieties of large degree

2.1. The statement

2.2. A Siegel type lemma

2.3. Multiplicity subspaces

2.4. The product theorem, part I

2.5. The product theorem, part II

2.6. The proof

3. The covering lemma

4. Collections of subsets

4.1. Base change and push forward

4.2. An example

4.3. Universally open morphisms

4.4. Flat families

5. Proof of Theorem A

\section{INTRODUCTION}

By a Fano variety we shall mean a smooth, connected, complex projective variety with ample anticanonical line bundle. In dimensions one and two, the only Fano varieties are the projective line and the Del Pezzo surfaces. In dimension three, there are already 104 distinct families of Fano varieties. This is a consequence of the classification of Fano threefolds, due to Fano, Iskovskikh, Mori, Mukai, and Shokurov (see [I1-4, MoMu, Mu] and the references therein). For Fano varieties of arbitrary dimension $m$, we have the following well-known conjectures.

Received by the editors September 27, 1990 and, in revised forms, December 20, 1990 and August 1, 1991.

1991 Mathematics Subject Classification. Primary 14J10.

Research partially supported by NSF Grant DMS-89-07710. 
Conjecture $\left(F_{m}\right)$. There exists a constant $C(m)$ such that $c_{1}(M)^{m} \leq C(m)$ for every $m$-dimensional Fano variety $M$.

Conjecture $\left(F_{m}^{\prime}\right)$. There are only finitely many deformation types of $m$-dimensional Fano varieties.

These two conjectures are equivalent. The implication $\left(F_{m}\right) \Rightarrow\left(F_{m}^{\prime}\right)$ follows from [KMa, Ma], while the reverse implication is trivial. For $m \leq 3$, these conjectures follow from the classification of Del Pezzo surfaces and Fano threefolds.

In this paper we shall establish these conjectures in the case of arbitrarydimensional Fano varieties with Picard number one. ${ }^{1}$ If $M$ is any Fano variety, we define $d(M)$ to be the least positive integer $d$ for which $M$ can be covered by rational curves of (anticanonical) degree at most $d$. By Mori's theory, $2 \leq d(M) \leq \operatorname{dim} M+1$ (see $\S 1$ ). Our main result is as follows.

Theorem A. For any Fano variety $M$ with Picard number one, $c_{1}(M)^{m} \leq$ $(m d)^{m}$, where $m=\operatorname{dim} M$ and $d=d(M)$.

Corollary. In each dimension, there are only finitely many deformation types of Fano varieties with Picard number one.

Kollár, Miyaoka, and Mori [KMiMo] have found a different and independent proof of Theorem A that is based on rational connectedness. They show that in a Fano variety with Picard number one, two general points can be joined by an irreducible rational curve of degree at most $m d(M)$. Both proofs of Theorem A involve the interaction between rational curves of low degree on the one hand, and multiplicity sets of plurianticanonical sections on the other hand; their proof focuses primarily on rational curves, while ours focuses primarily on multiplicity sets. In this sense, the two approaches are dual to one another. There is also a differential-geometric approach to this problem due to Tsuji.

The basic idea of our proof is very simple. Let $M$ be a Fano variety, and assume that $c_{1}(M)^{m}>(m d)^{m}$, where $m=\operatorname{dim} M$ and $d=d(M)$. We would like to obtain a contradiction, at least if $M$ has Picard number one. Let $f: \mathbf{P}^{1} \rightarrow M$ be a morphism of degree $d$, and set $p=f(0)$. Because of its large degree, $M$ admits many plurianticanonical sections. A dimension-counting argument shows that some plurianticanonical section must vanish to very high order at $p$. Specifically, there exists an integer $\nu>0$ and a section $s \in$ $H^{0}\left(M, K_{M}^{-\nu}\right)-\{0\}$ whose vanishing order at $p$ exceeds $m d \nu$. Now consider the pulled-back section $f^{*} s \in H^{0}\left(\mathbf{P}^{1}, f^{*} K_{M}^{-\nu^{\prime}}\right)$. On the one hand, the vanishing order of $f^{*} s$ at 0 exceeds $m d \nu$. On the other hand, the line bundle $f^{*} K_{M}^{-\nu}$ has degree only $d \nu$. This forces $f^{*} s$ to be identically zero. Thus the image $f\left(\mathbf{P}^{1}\right)$ must lie entirely inside the zero set of $s$. We extend these ideas by proving a product theorem that is analogous to Faltings's product theorem (see

\footnotetext{
${ }^{1}$ It should be noted that the Picard number of a Fano variety is always equal to the second Betti number, and is therefore a topological invariant.
} 
[F]). The product theorem implies that $M$ can be covered by proper subvarieties $V \subset M$ that satisfy the following condition: For any morphism $g: \mathbf{P}^{1} \rightarrow M$ with $g^{*} T_{M}$ semipositive of degree $d$, either $g\left(\mathbf{P}^{1}\right) \cap V=\varnothing$ or else $g\left(\mathbf{P}^{1}\right) \subset V$. These $V$ 's essentially form an algebraic family, a suitable subfamily of which will sweep out a closed hypersurface $H \subset M$. Finally, for any morphism $g$ : $\mathbf{P}^{1} \rightarrow M$ with $g^{*} T_{M}$ semipositive of degree $d$ and $g\left(\mathbf{P}^{1}\right) \not \subset H, g\left(\mathbf{P}^{1}\right) \cap H=\varnothing$. Thus $M$ contains a curve and a hypersurface that are mutually disjoint. This forces the Picard number of $M$ to be at least two.

In an earlier version [N2] of the present paper, I gave a proof of Theorem A in the four-dimensional case. That proof was already independent of dimension except near the end, and here I adapt that proof to all dimensions by simplifying and improving the argument near the end, following a suggestion by J. Kollár [K2], to whom I am greatly indebted. Another improvement is as follows. The main intermediate theorem (Theorem 1 in the present paper) had appeared already in [N2], but the proof given there depended on transcendental techniques from the study of existence of Kähler-Einstein metrics on Fano varieties (see [N3-6]). The proof given here is entirely algebro-geometric in nature and is motivated by Faltings's work on diophantine approximation (see $[\mathrm{F}]$ ).

\section{Rational CURVES}

1.1. Deformation theory of morphisms. The reader is referred to Mori's paper [Mo] for the theory of deformation of morphisms between smooth projective varieties. We very informally recall here several relevant points. Let $M$ be a smooth projective variety. The space $\operatorname{Maps}\left(\mathbf{P}^{1}, M\right)$ of all morphisms $f$ : $\mathbf{P}^{1} \rightarrow M$ has countably many connected components, each of which has the natural structure of a quasi-projective scheme. There is a universal morphism $\mathbf{P}^{1} \times \operatorname{Maps}\left(\mathbf{P}^{1}, M\right) \rightarrow M$. The Zariski tangent space of $\operatorname{Maps}\left(\mathbf{P}^{1}, M\right)$ at $f$ is isomorphic to $H^{0}\left(M, f^{*} T_{M}\right)$. If $H^{1}\left(M, f^{*} T_{M}\right)=0$ then $\operatorname{Maps}\left(\mathbf{P}^{1}, M\right)$ is smooth at $f$ and there are no obstructions to deforming $f$.

1.2. Free rational curves. Let $M$ be a smooth projective variety. Grothendieck's splitting theorem asserts that every holomorphic vector bundle on $\mathbf{P}^{1}$ is isomorphic to a direct sum of line bundles. Given any morphism $f: \mathbf{P}^{1} \rightarrow M$, we can thus write $f^{*} T_{M} \cong \mathscr{O}_{\mathbf{P}^{1}}\left(a_{1}\right) \oplus \cdots \oplus \mathscr{O}_{\mathbf{P}^{1}}\left(a_{m}\right)$ for suitable (uniquely determined) integers $a_{1} \geq \cdots \geq a_{m}$, where $m=\operatorname{dim} M$. By regarding the differential $D f$ as a global holomorphic section of $f^{*} T_{M} \otimes T_{\mathbf{P}^{1}}^{-1} \cong \mathscr{O}_{\mathbf{P}^{1}}\left(a_{1}-2\right) \oplus \cdots \oplus \mathscr{O}_{\mathbf{P}^{1}}\left(a_{m}-2\right)$, we see that $a_{1} \geq 2$ (unless $f$ is constant). The number $a_{1}+\cdots+a_{m}$ will be called the (anticanonical) degree of $f$. It is equal to the degree of the line bundle $f^{*} K_{M}^{-1}$ on $\mathbf{P}^{1}$.

Proposition 1. The following conditions on $f$ are equivalent:

(F1) $f^{*} T_{M}$ is semipositive (i.e., $a_{m} \geq 0$ ).

(F2) $h^{1}\left(\mathbf{P}^{1}, f^{*} T_{M} \otimes \mathscr{O}_{\mathbf{P}^{1}}(-1)\right)=0$.

(F3) $f^{*} T_{M}$ is generically generated by global sections. 
The proof is very easy and is omitted. When these conditions hold we shall say that $f$ is free. Note that freeness is a Zariski-open condition with respect to deformation (by the semicontinuity theorem and (F2)) .

The following application of Sard's theorem will be used to deduce the existence of free rational curves. This type of result fails in characteristic $p$, where there are examples of uniruled surfaces of general type.

Proposition 2. Suppose that (for some k) $F: \mathbf{P}^{1} \times \Delta^{k} \rightarrow M$ is a morphism whose image has positive measure in $M$. Then $F(*, t)$ will be free for some, and hence for general, $t$. (Here and henceforth, $\Delta$ denotes the unit disk.)

Proof. By Sard's theorem, the morphism $F$ is generically submersive. The holomorphic tangent bundle of $\mathbf{P}^{1} \times \Delta^{k}$ is generated by global sections. Thus $F^{*} T_{M}$ is generically generated by global sections. Clearly $F(*, t)$ will satisfy (F3) for some $t$.

1.3. The invariant $d(M)$. We continue to work over the complex numbers. For any Fano variety $M$ we definite integers $d_{1}(M), d_{2}(M)$ as follows.

(1) Define $d_{1}(M)$ to be the least positive integer $d$ for which there exists a morphism $F: \mathbf{P}^{1} \times \Delta^{k} \rightarrow M$ (for some $k$ ) whose image has positive measure in $M$, and such that each morphism $F(*, t)$ has degree $d$.

(2) Define $d_{2}(M)$ to be the least positive integer $d$ for which there exists a morphism $\mathbf{P}^{1} \rightarrow M$ that is free of degree $d$.

We shall show that $d_{1}(M)=d_{2}(M)$. Proposition 2 implies that $d_{2}(M) \leq$ $d_{1}(M)$. For the reverse inequality, suppose that $f: \mathbf{P}^{1} \rightarrow M$ is free of degree $d$ for some $d>0$. Because $f$ is free, it can be deformed without obstruction, and there exists (for suitable $k$ ) a holomorphic submersion $F: \mathbf{P}^{1} \times \Delta^{k} \rightarrow$ $M$ such that $F(*, 0)=f(*)$. The inverse function theorem ensures that the image of $F$ will be open and will thus have positive measure. This shows that $d_{1}(M) \leq d_{2}(M)$, as desired.

Denote by $d(M)$ the common value of $d_{1}(M)$ and $d_{2}(M)$. We shall show that $2 \leq d(M) \leq \operatorname{dim} M+1$. By the methods of Mori (see [K1, Mo]) we know that $M$ can be covered by rational curves whose degrees do not exceed $\operatorname{dim} M+$ 1. By standard Hilbert scheme arguments we can then obtain a morphism $F: \mathbf{P}^{1} \times \Delta^{k} \rightarrow M$ whose image in $M$ has positive measure, and such that each morphism $F(*, t)$ is nonconstant of degree at most $\operatorname{dim} M+1$. Thus $d_{1}(M) \leq \operatorname{dim} M+1$. On the other hand, it is easy to see that $d_{2}(M) \geq 2$, and the assertion follows.

\section{SPECIAL SUbVARIETIES IN FANO VARIETIES OF LARGE DEGREE}

2.1. The statement. In this section we shall prove the following theorem.

Theorem 1. Let $M$ be an $m$-dimensional Fano variety, and let $d$ be a positive integer. Assume that $c_{1}(M)^{m}>(m d)^{m}$. Then there exists a collection $\mathscr{V}$ of 
proper irreducible subvarieties of $M$ satisfying the following conditions:

(V1) $M$ is covered by the subvarieties in $\mathscr{V}$; that is, for each point $p \in M$ there exists at least one $V \in \mathscr{V}$ such that $p \in V$.

(V2) For any morphism $f: \mathbf{P}^{1} \rightarrow M$ that is free of degree $d$, and any $V \in \mathscr{V}$, either $f\left(\mathbf{P}^{1}\right) \subset V$ or $f\left(\mathbf{P}^{1}\right) \cap V=\varnothing$.

Remarks. (1) By a proper subvariety we mean a subvariety that is nonempty and not equal to the entire ambient space.

(2) Let $M$ be a Fano variety, and set $m=\operatorname{dim} M$ and $d=d(M)$. Consider the following equivalence relation on the points of $M$. Two points are related if and only if they can be joined by a chain of free rational curves of degree $d$. The theorem above implies that if $c_{1}(M)^{m}>(m d)^{m}$ then no equivalence class can be Zariski-dense. On the other hand, in [KMiMo] it is shown that if $M$ has Picard number one then two general points can be joined by a chain of free rational curves of degree $d$. This gives another proof of Theorem $\mathrm{A}$ that extrapolates between the proof in [KMiMo] and the proof in the present paper.

The remaining subsections will be devoted to proving this theorem. Various themes from diophantine approximation will be apparent throughout. In particular, our product theorem is similar to G. Faltings's product theorem [F].

\subsection{A Siegel type lemma.}

Lemma 1. Let $M$ be an m-dimensional smooth connected projective variety, and let $L$ be an ample line bundle on $M$. For every point $p \in M$ and every positive real number $A<\left(c_{1}(L)^{m}\right)^{1 / m}$, there exists an integer $\nu>0$ and a section $s \in H^{0}\left(M, L^{\nu}\right)-\{0\}$ whose vanishing order at $p$ exceeds $\nu A$.

Proof of Lemma 1. By Riemann-Roch and Serre vanishing we have

$$
h^{0}\left(M, L^{\nu}\right) \geq \frac{1}{m !} c_{1}(L)^{m} \nu^{m}+O\left(\nu^{m-1}\right)
$$

as $\nu \rightarrow \infty$. On the other hand,

$$
h^{0}\left(M, \mathscr{O}_{M} / \mathscr{I}^{\eta+1}\right)=\left(\begin{array}{c}
m+\eta \\
m
\end{array}\right) \leq \frac{1}{m !} \eta^{m}+O\left(\eta^{m-1}\right)
$$

as $\eta \rightarrow \infty$. Here $\mathscr{I}$ denotes the ideal sheaf of the reduced point $p$. Now set $\eta=[\nu A]$ for some $\nu>>0$. Here the brackets denote the greatest integer function. The above inequalities, together with the inequality $A<\left(c_{1}(M)^{m}\right)^{1 / m}$, imply that $h^{0}\left(M, L^{\nu}\right)>h^{0}\left(M, \mathscr{O}_{M} / \mathscr{I}^{\eta+1}\right)$. Hence there exists a section $s \in H^{0}\left(M, L^{\nu}\right)-\{0\}$ whose Taylor series expansion of order $\eta$ at $p$ is trivial.

2.3. Multiplicity subspaces. Let $f$ be a holomorphic function on a domain $\Omega \subset \mathbf{C}^{m}$. For each integer $j$, define $V_{j}(f) \subset \Omega$ to be the set of all points at which the vanishing order of $f$ is at least $j$. We can endow $V_{j}(f) \subset \Omega$ with the natural structure of a complex analytic subspace, possibly nonreduced, by 
taking as a defining ideal the ideal generated by all partial derivatives of $f$ of order strictly less than $j$. However, we will usually regard $V_{j}(f) \subset \Omega$ as merely a subvariety, ignoring any nonreduced structure. We set $V_{j}(f)=\Omega$ for $j \leq 0$.

These concepts globalize in the obvious way. Let $M$ be a complex manifold, let $L$ be a holomorphic line bundle on $M$, and let $s \in H^{0}(M, L)$. For each integer $j$, we can define a complex analytic subspace $V_{j}(s) \subset M$ consisting of precisely those points at which the vanishing order of $s$ is at least $j$.

An alternative approach for showing that $V_{j}(s)$ has a natural complex analytic subspace structure uses jets directly rather than partial derivatives. It has the advantage of working in characteristic $p$ and on singular spaces; however, the present approach will be adequate for our purposes.

Now let $f: M \rightarrow N$ be a holomorphic mapping of complex manifolds, and let $s$ be a global holomorphic section of a holomorphic line bundle on $N$. Then $f^{*} V_{j}(s) \subset V_{j}\left(f^{*} s\right)$, with equality if $f$ is submersive.

\subsection{The product theorem, part $I$.}

Theorem 2. Fix positive integers $d$ and $k$. Set $M=\mathbf{P}^{1} \times \Delta^{k}$, let $L$ be a holomorphic line bundle on $M$ whose restriction to $\mathbf{P}^{1} \times\{0\}$ has degree $d$, and let $s \in H^{0}(M, L)$. For every integer $j$, either $\mathbf{P}^{1} \times\{0\} \cap V_{j+d}(s)=\varnothing$ or $\mathbf{P}^{1} \times\{0\} \subset V_{j}(s)$.

Proof. We may assume that $s$ is not identically zero, for otherwise we would be done. If $k>1$ then we can reduce to the case $k=1$ by restricting to a linearly embedded disk $\Delta \subset \Delta^{k}$ passing through the origin, in a sufficiently general direction. Thus we may assume that $k=1$ and that $M=\mathbf{P}^{1} \times \Delta$. Let $z$ denote the holomorphic coordinate function of $\Delta$, pulled back to $M$ via the projection. Note that the desired assertion holds for $s$ if and only if it holds for $z s$. Therefore, by dividing $s$ by a suitable power of $z$, we can reduce to the case in which $s$ does not vanish identically along the curve $\mathbf{P}^{1} \times\{0\}$. Since the restriction of $L$ to $\mathbf{P}^{1} \times\{0\}$ has degree $d$, the vanishing order of $s$ at each point in $\mathbf{P}^{1} \times\{0\}$ will not exceed $d$. Hence $\mathbf{P}^{1} \times\{0\} \cap V_{j+d}(s)$ will be nonempty only when $j \leq 0$, in which case $V_{j}(s)=M$, from which the desired conclusion follows.

\subsection{The product theorem, part II.}

Theorem 3. Let $M$ be a complex manifold, let $L$ be a holomorphic line bundle on $M$, and let $s \in H^{0}(M, L)$. Suppose $f: \mathbf{P}^{1} \rightarrow M$ is a morphism that is free, and set $d=\operatorname{deg}\left(f^{*} L\right)$. For every integer $j$, either $f\left(\mathbf{P}^{1}\right) \cap V_{j+d}(s)=\varnothing$ or $f\left(\mathbf{P}^{1}\right) \subset V_{j}(s)$.

Proof. Let us assume that $f(0) \in V_{j+d}(s)$. We want to show that $f\left(\mathbf{P}^{1}\right) \subset$ $V_{j}(s)$. We may assume that $f$ is nonconstant, for otherwise we would be done. Since $f$ is free, it can be deformed without obstruction, and there exists (for suitable $k$ ) a holomorphic submersion $F: \mathbf{P}^{1} \times \Delta^{k} \rightarrow M$ such that $F(*, 0)=$ 
$f(*)$. The submersivity implies that $F^{*} V_{j+d}(s)=V_{j+d}\left(F^{*} s\right)$. Since $(0,0) \in$ $F^{*} V_{j+d}(s)=V_{j+d}\left(F^{*} s\right)$, we can deduce from $\S 2.4$ that $\mathbf{P}^{1} \times\{0\} \subset V_{j}\left(F^{*} s\right)=$ $F^{*} V_{j}(s)$. The result follows.

2.6. The proof. In this subsection we shall complete the proof of Theorem 1 . Let $m, d$, and $M$ be as in the statement of the theorem. Fix any point $p \in M$. Since $c_{1}(M)^{m}>(m d)^{m}$, we can apply Lemma 1 to get an integer $\nu>0$ and a section $s \in H^{0}\left(M, K_{M}^{-\nu}\right)-\{0\}$ whose vanishing order at $p$ strictly exceeds $m d \nu$.

For simplicity of notation, we set $W_{j}=V_{1+d \nu j}(s)$. We have a descending chain of subvarieties

$$
W_{0} \supset W_{1} \supset \cdots \supset W_{m} \ni p .
$$

For each $j \in\{0, \ldots, m\}$ we choose an irreducible component of $W_{j}$ containing $p$, and denote this irreducible component by $W_{j}^{\prime}$. We may assume that these irreducible components have been chosen so that

$$
W_{0}^{\prime} \supset W_{1}^{\prime} \supset \cdots \supset W_{m}^{\prime} \ni p .
$$

Since this chain has length greater than the dimension of $M$, there exists some $j \in\{0, \ldots, m-1\}$ such that

$$
W_{j}^{\prime}=W_{j+1}^{\prime}
$$

Set $W:=W_{j}^{\prime}=W_{j+1}^{\prime}$. Clearly $p \in W$, and it remains only to verify that condition (V2) is satisfied in an appropriate sense. Namely, suppose that $f$ : $\mathbf{P}^{1} \rightarrow M$ is any free morphism of degree $d$ with $f(0) \in W$. We must show that $f\left(\mathbf{P}^{1}\right) \subset W$; then the theorem will be proved.

Since $f(0) \in W_{j+1}$, we can deduce from $\S 2.5$ that $f\left(\mathbf{P}^{1}\right) \subset W_{j}$. Recall that $W$ is an irreducible component of $W_{j}$. For most points $q \in W$, the variety $W_{j}$ will be irreducible locally at $q$ and will agree with $W$ in an open neighborhood of $q$. For some special points $q \in W$, however, the variety $W_{j}$ may be reducible locally at $q$, in which case we will have to be careful.

First consider the case in which the point $f(0) \in W$ is one at which $W_{j}$ is locally irreducible. In this case, we can deduce from $f\left(\mathbf{P}^{1}\right) \subset W_{j}$ that $f\left(\mathbf{P}^{1}\right) \subset$ $W$, as desired. Next consider the case in which the point $f(0) \in W$ is one at which $W_{j}$ is locally reducible. Deform $f$ slightly to get a free morphism $g: \mathbf{P}^{1} \rightarrow M$ such that $g(0) \in W$ and such that $W_{j}$ is locally irreducible at $g(0)$. From our argument in the first case we are able to conclude that $g\left(\mathbf{P}^{1}\right) \subset W$. Finally, let $g$ converge back to $f$ to get $f\left(\mathbf{P}^{1}\right) \subset W$, as desired. This concludes the proof.

\section{THE COVERING LEMMA}

Lemma 2. Let $M$ be a complete irreducible variety, and let $V \subset M \times T$ be a family of proper subschemes of $M$ parameterized by a projective scheme $T$. 
Suppose that $M$ is the union of all the $V_{t}$. Then there exists a closed hypersurface in $M$ that is the union of some of the $V_{t}$.

Remarks. (1) By a proper subscheme of $M$ we mean a subscheme that is nonempty and not equal to all of $M$.

(2) We do not require $V$ to be flat over $T$.

Proof. Obviously $T$ cannot be zero-dimensional. First consider the case in which $\operatorname{dim} T=1$. We will complete the proof in this case by showing that some $V_{t}$ is already a hypersurface in $M$. We have $\operatorname{dim} V \leq \max _{t} \operatorname{dim} V_{t}+\operatorname{dim} T$. We also have $\operatorname{dim} M \leq \operatorname{dim} V$, since $M$ is covered by the $V_{t}$. Thus $\operatorname{dim} V_{t} \geq$ $\operatorname{dim} V-\operatorname{dim} T \geq \operatorname{dim} M-1$ for some $t$, as desired.

Now suppose that $\operatorname{dim} T \geq 2$. Consider a Lefschetz pencil of hypersurfaces of $T$. This consists of an irreducible subvariety $\Lambda \subset T \times \mathbf{P}^{1}$ such that each $\Lambda_{s}\left(s \in \mathbf{P}^{1}\right)$ is a hypersurface in $T$ and such that $T$ is the union of all these hypersurfaces. For each $s \in \mathbf{P}^{1}$, define a subvariety $W_{s} \subset M$ as follows. Set theoretically, $W_{s}=\bigcup_{t \in \Lambda_{s}} \Sigma_{t}$. These subvarieties $W_{s}$ can be given scheme structures so that they fit together to form an algebraic family of subschemes of $M$ with parameter space $\mathbf{P}^{1}$. Since the parameter space is one-dimensional, we can apply the argument from the paragraph above to conclude that some $W_{s}$ is a hypersurface in $M$, in which case we are done, or is all of $M$, in which case we can replace $T$ by some hypersurface in $T$ and proceed by induction on $\operatorname{dim} T$.

\section{Collections of subsets}

4.1. Base change and push forward. Let $\mathscr{C}$ be a collection of subsets of an algebraic variety $M$. For any algebraic variety $T$, define $\mathscr{C}_{T}$, a collection of subsets of $M \times T$, as follows. A subset $X \subset M \times T$ belongs to $\mathscr{C}_{T}$ if and only if $X_{t} \subset M$ belongs to $\mathscr{C}$, for every $t \in T$. (Here $X_{t}$ denotes the projection of $X \cap(M \times\{t\}) \subset M \times T$ to $M$.)

The collection $\mathscr{C}$ may (or may not) satisfy one or more of the following properties: (1) closure under finite unions, (2) closure under arbitrary unions, (3) closure under finite intersections, (4) closure under arbitrary intersections, (5) closure under complements, (6) possession of the empty subset, and (7) closure under Zariski-closure (i.e., for every subset $X \subset M$ belonging to $\mathscr{C}$, the Zariski-closure $\bar{X} \subset M$ also belongs to $\mathscr{C}$ ). We shall say that $\mathscr{C}$ satisfies a property $P$ universally if $\mathscr{C}_{T}$ satisfies $P$ for every algebraic variety $T$.

Thus we shall say that $\mathscr{C}$ is universally closed under Zariski-closure, abbreviated u.c.u.z.c., if the following is true. For any algebraic variety $T$, and any subset $X \subset M \times T$ belonging to $\mathscr{C}_{T}$, the Zariski-closure $\bar{X} \subset M \times T$ also belongs to $\mathscr{C}_{T}$.

Given a morphism of varieties $f: M \rightarrow N$, define the push-forward $f_{*} \mathscr{C}$, a collection of subsets of $N$, as follows. A subset $X \subset N$ belongs to $f_{*} \mathscr{C}$ if and only if its inverse image $f^{-1}(X) \subset M$ belongs to $\mathscr{C}$. 
4.2. An example. Let $S$ be any algebraic variety, and set $M=\mathbf{P}^{1} \times S$. Let $\mathscr{C}$ be the collection of all subsets of $M$ of the form $\mathbf{P}^{1} \times X$, where $X$ is an arbitrary subset of $S$. Obviously $\mathscr{C}$ satisfies properties (1)-(6) above. We shall show that $\mathscr{C}$ satisfies (7) and is u.c.u.z.c. Let $G$ be the group of all automorphisms of $\mathbf{P}^{1}$. Let $G$ act on $M$ by acting as usual on the first factor $\mathbf{P}^{1}$ and acting trivially on the second factor $S$. Then $\mathscr{C}$ consists of precisely those subsets of $M$ that are invariant under $G$. If a given subset of $M$ is invariant under $G$, then its Zariski-closure will also be invariant under $G$, since the action of $G$ on $M$ is an algebraic one. This shows that $\mathscr{C}$ is closed under Zariski-closure. That $\mathscr{C}$ is u.c.u.z.c. can be shown in a similar fashion. Suppose that $T$ is any algebraic variety. Let $G$ act on $M \times T$ by acting as before on the first factor $M$, and acting trivially on the second factor $T$. Then $\mathscr{C}_{T}$ consists of precisely those subsets of $M \times T$ that are invariant under $G$. This shows that $\mathscr{C}_{T}$ is closed under Zariski-closure, and hence that $\mathscr{C}$ itself is u.c.u.z.c.

4.3. Universally open morphisms. A morphism of algebraic varieties $f: M \rightarrow$ $N$ is said to be open if it takes Zariski-open sets to Zariski-open sets. It is said to be universally open if the morphism $f \times \mathrm{Id}_{T}: M \times T \rightarrow N \times T$ is open, for every algebraic variety $T$.

Example. A flat morphism of algebraic varieties is universally open.

Lemma 3. If $f: M \rightarrow N$ is an open morphism of varieties then $f^{-1}(\bar{X})=$ $\overline{f^{-1}(X)}$ for every subset $X \subset N$. (Here the bar denotes Zariski-closure.)

Proof. Clearly $f^{-1}(\bar{X}) \supset \overline{f^{-1}(X)}$. For the reverse inclusion, set $U=M-$ $\overline{f^{-1}(X)}$. Since $f$ is an open morphism, $f(U)$ will be Zariski-open in $N$. Clearly $f(U) \cap X=\varnothing$, and hence $f(U) \cap \bar{X}=\varnothing$, and hence $U \cap f^{-1}(\bar{X})=\varnothing$. This shows that $f^{-1}(\bar{X}) \subset \overline{f^{-1}(X)}$, completing the proof.

Proposition 3. Let $f: M \rightarrow N$ be a morphism of varieties that is universally open, and let $\mathscr{C}$ be a collection of subsets of $M$ that is u.c.u.z.c. Then $f_{*} \mathscr{C}$ is u.c.u.z.c.

Proof. For any algebraic variety $T$, we know that $\mathscr{C}_{T}$ is closed under Zariskiclosure and that $f \times \operatorname{Id}_{T}: M \times T \rightarrow N \times T$ is open. The lemma above implies that $\left(f \times \operatorname{Id}_{T}\right)_{*} \mathscr{C}$ is closed under Zariski-closure. Since $\left(f \times \operatorname{Id}_{T}\right)_{*} \mathscr{C}=(f \mathscr{C})_{T}$, the latter is also closed under Zariski-closure, which is what we want to show.

\subsection{Flat families.}

Proposition 4. Let $\mathscr{C}$ be a collection of subsets of an algebraic variety $M$, and let $V \subset M \times T$ be a flat family of subschemes of $M$, parameterized by a scheme $T$. Define $T^{\prime} \subset T$ to be the set of all $t \in T$ for which $V_{t}$ (as a subset of $M$, ignoring all scheme structure) belongs to $\mathscr{C}$. Assume that $\mathscr{C}$ is u.c.u.z.c. and contains the empty set. Then $T^{\prime}$ is Zariski-closed in $T$. 
Proof. We may assume that $T^{\prime}$ is Zariski-dense in $T$. Our goal is to show that $T^{\prime}=T$ or equivalently, that $V \in \mathscr{C}_{T}$. Let $W=V \cap\left(M \times T^{\prime}\right) \subset M \times T$. Clearly $W \in \mathscr{C}_{T}$, since $W_{t}=V_{t} \in \mathscr{C}$ for $t \in T^{\prime}$ and $W_{t}=\varnothing \in \mathscr{C}$ for $t \in T-T^{\prime}$. We know that $\mathscr{C}_{T}$ is closed under Zariski-closure, since $\mathscr{C}$ is u.c.u.z.c., by hypothesis. Therefore, $\bar{W} \in \mathscr{C}_{T}$. Clearly $\bar{W} \subset V$. Now set $U=V-\bar{W}$, to be regarded as a Zariski-open subset of $V$. Since the projection $p: V \rightarrow T$ is flat by assumption, it is open, whence the image $p(U)$ is Zariski-open in $T$. Since $p(U)$ is disjoint from the Zariski-dense subset $T^{\prime} \subset T$, it is empty. Therefore, $\bar{W}=V$, whence $V \in \mathscr{C}_{T}$, as desired.

\section{Proof of Theorem A}

In this section we shall prove Theorem $\mathrm{A}$. Suppose that $M$ is a Fano variety with $c_{1}(M)^{m}>(m d)^{m}$, where $m=\operatorname{dim} M$ and $d=d(M)$. We wish to show that the Picard number of $M$ is strictly greater than one.

5.1. Let $S$ be the (nonempty) collection of all morphisms $f: \mathbf{P}^{1} \rightarrow M$ that are free of degree $d$. We know from Hilbert scheme theory that $S$ has the natural structure of a quasi-projective scheme, and that there exists a universal morphism $F: \mathbf{P}^{1} \times S \rightarrow M$.

5.2. Observe that $S$ is a smooth everywhere and $F$ is submersive. The first statement is a consequence of the fact that each $f: \mathbf{P}^{1} \rightarrow M$ in our collection is free and thus satisfies $H^{1}\left(\mathbf{P}^{1}, f^{*} T_{M}\right)=0$. The second statement is a consequence of the following two facts. First, the linear mapping $T_{S, f} \rightarrow H^{0}\left(\mathbf{P}^{1}, f^{*} T_{M}\right)$ is surjective since $F$ is everywhere a universal deformation. Second, the bundle $f^{*} T_{M}$ is generated by its global sections since it is semipositive.

Because the morphism $F$ is submersive, it is flat, and hence universally open,

5.3. Let $\mathscr{C}$ be the collection of all subsets of $\mathbf{P}^{1} \times S$ of the form $\mathbf{P}^{1} \times X$, where $X$ is an arbitrary subset of $S$. Obviously $\mathscr{C}$ contains the empty subset and is closed under arbitrary unions. We have seen that $\mathscr{C}$ is universally closed under Zariski-closure (u.c.u.z.c.). See $\S 4.2$ for an elaboration on this.

5.4. Denote by $F_{*} \mathscr{C}$ the collection of all subsets $X \subset M$ for which $F^{-1}(X) \in$ $\mathscr{C}$. The following description of $F_{*} \mathscr{C}$ may be helpful. A subset $X \subset M$ belongs to $F_{*} \mathscr{C}$ if and only if the following is true. For every morphism $f$ : $\mathbf{P}^{1} \rightarrow M$ that is free of degree $d$, either $f\left(\mathbf{P}^{1}\right) \subset X$ or $f\left(\mathbf{P}^{1}\right) \cap S=\varnothing$.

5.5. Since $\mathscr{E}$ contains the empty subset and is closed under arbitrary unions, the same will be true of $F_{*} \mathscr{C}$. Since $\mathscr{C}$ is u.c.u.z.c. and the morphism $F$ is universally open, $F_{*} \mathscr{C}$ will also be u.c.u.z.c. (see Proposition 3 ).

5.6. We now come to a key idea of the proof. Suppose for the time being that we have an algebraic family $\left\{V_{t}\right\}$ of proper subschemes of $M$, with projective parameter space $T$, such that each $V_{t}$ belongs (as a subset of $M$, ignoring all 
subscheme structure) to $F_{*} \mathscr{C}$. Then we can use the covering lemma (Lemma 2) to construct an algebraic hypersurface $H \subset M$ that is the union of some of the $V_{t}$. This hypersurface will belong to $F_{*} \mathscr{C}$ since the latter is closed under arbitrary unions. Thus for every $s \in S$ we will have eithere $F\left(\mathbf{P}^{1}, s\right) \subset H$ or $F\left(\mathbf{P}^{1}, s\right) \cap H=\varnothing$. Since $F$ is an open morphism, it cannot satisfy $F\left(\mathbf{P}^{1} \times S\right) \subset$ $H$. Thus there exists some $s \in S$ for which $F\left(\mathbf{P}^{1}, s\right) \cap H=\varnothing$. In particular, $M$ contains a curve and a hypersurface that are mutually disjoint. This shows that the Picard number of $M$ must be at least two, as desired.

5.7. We now turn our attention to constructing an algebraic family of subschemes as above. According to Theorem 1, $M$ can be covered by proper algebraic subvarieties that belong to $F_{*} \mathscr{C}$. It is here that the hypothesis $c_{1}(M)^{m}>$ $(m d)^{m}$ has been used. We want to show that these subvarieties can be put together to form an algebraic family.

5.8. Let $\operatorname{Hilb}(M)$ be the Hilbert scheme parameterizing all proper subschemes of $M$. Let $T \subset \mathrm{Hilb}(M)$ be the set of Hilbert points of all proper subschemes of $M$ that (as subsets of $M$ ) belong to $F_{*} \mathscr{C}$. Recall that $\operatorname{Hilb}(M)$ is a countable disjoint union of connected projective schemes. Let $\operatorname{Hilb}(M)_{1}$, $\operatorname{Hilb}(M)_{2}, \ldots$ be an enumeration of these connected components of $\operatorname{Hilb}(M)$. Set $T_{j}=\operatorname{Hilb}(M)_{j} \cap T(j=1,2, \ldots)$. Since $F_{*} \mathscr{C}$ contains the empty set and is u.c.u.z.c. (see $\S 5.5$ ), each $T_{j}$ will be Zariski-closed in $\operatorname{Hilb}(M)_{j}$ (see Proposition 4).

5.9. Each $T_{j}$ can be endowed with the induced reduced scheme structure and regarded as the parameter space of an algebraic family of subschemes of $M$. Together these families cover all of $M$ (by $\S 5.7$ ). Since $M$ cannot be expressed as a countable union of lower-dimensional subvarieties, at least one of these families must already cover all of $M$. We can then proceed as in $\S 5.6$ to conclude that the Picard number of $M$ must be at least two. This concludes our proof of Theorem A.

Remarks. (1) In $\S 5.6$ we do not really make full use of the hypothesis concerning the Picard number of $M$. The hypersurface $H \subset M$ can be taken to pass through a general point. This is enough to get the desired contradiction even for some $M$ with Picard number greater than one

(2) In $\S 5.9$ all we really need is that $M$ cannot be expressed as a finite union of lower-dimensional subvarieties. Indeed, the subvarieties $V \subset M$ constructed in Theorem 1 will form a bounded collection.

\section{ACKNOWLedgment}

The results and methods of this paper were presented in [N1]. I would like to thank J. Kollár and Y.-T. Siu for helpful suggestions and encouragement. I would also like to thank D. Barlet, F. Bogomolov, J.-M. Hwang, S. Kleiman, Y. Manin, Y. Miyaoka, S. Mori, S. Mukai, H. Tsuji, S. K. Yeung, and the many others who have contributed to my understanding of the subject. 


\section{REFERENCES}

[F] G. Faltings, Diophantine approximation on abelian varieties, preprint.

[I1] V. A. Iskovskikh, Fano 3-folds. I, Math. USSR-Izv. 11 (1977), 485-527.

[I2] _ Fano 3-folds. II, Math. USSR-Izv. 11 (1978), 469-506.

[13] _ Anticanonical models of three-dimensional algebraic varieties, J. Soviet Math. 13 (1980), 745-814; (1978), 469-506.

[I4] _ Double projection from a line on Fano threefolds of the first species, Math. USSR-Sb. 66 (1) (1990), 265-284.

[K1] J. Kollár, Fano varieties of large index, Vestnik Moskov Gos. Univ. (1981), 31-34. (Russian)

[K2] - private communication.

[KMiMo] J. Kollár, Y. Miyaoka, and S. Mori, in preparation.

[KMa] J. Kollár and T. Matsusaka, Riemann-Roch type inequalities, Amer. J. Math. 105 (1983), 229-252.

[Ma] T. Matsusaka, Polarised varieties with given Hilbert polynomial, Amer. J. Math. 94 (1972), 1027-1077.

[Mo] S. Mori, Projective manifolds with ample tangent bundles, Ann. of Math. 110 (1979), 593606.

[MoMu] S. Mori and S. Mukai, Classification of Fano threefolds with $B_{2} \geq 2$, Manuscripta Math. 39 (1981), 147-162.

[Mu] S. Mukai, Biregular classification of Fano 3-folds and Fano manifolds of coindex 3, Proc. Nat. Acad. Sci. U.S.A. 86 (1989), 3000-3002.

[N1] A. M. Nadel, lecture at the JAMI Conference (The Johns Hopkins Unversity, November 7, 1990); lecture at the joint Los Angeles-Salt Lake City Conference on Algebraic Geometry (Salt Lake City, November 17, 1990); lecture at the Osaka International Conference on Complex Geometry (Osaka, December 12, 1990).

[N2] _ A finiteness theorem for Fano 4-folds, Massachusetts Institute of Technology, Cambridge, MA, preprint, 1990.

[N3] _ Multiplier ideal sheaves and existence of Kähler-Einstein metrics of positive scalar curvature, Proc. Nat. Acad. Sci. U.S.A. 86 (1989), 7299-7300.

[N4] _ Multiplier ideal sheaves and Kähler-Einstein metrics of positive scalar curvature, Ann. of Math. (2) 132 (1990), 549-596.

[N5] - The behavior of multiplier ideal sheaves under morphisms, Massachusetts Institute of Technology, Cambridge, MA, preprint 1989.

[N6] _- An observation concering the existence of Kähler-Einstein metrics on Del Pezzo surfaces, Massachusetts Institute of Technology, Cambridge, MA, preprint, 1.990.

Department of Mathematics, Massachusetts Institute of Technology, Cambridge, MASSACHUSETTS 02139 